\title{
Effects of Temperature on the Rheological Behavior of Worm-like Micelles in a Mixed Nonionic Surfactant System
}

\author{
Kaname Hashizaki* , Hiroyuki Taguchi and Yoshihiro Saito \\ College of Pharmacy, Nihon University (7-7-1 Narashinodai, Funabashi, Chiba 274-8555, JAPAN)
}

\begin{abstract}
We examined the effects of temperature on the rheological behavior of worm-like micelles in a nonionic surfactant system consisting of polyoxyethylene (10) phytosterol $\left(\right.$ PhyEO $\left._{10}\right) /$ glyceryl monocaprylate $\left(\right.$ GFA-C 8 )/Water. First, the phase diagram of a PhyEO $_{10} /$ GFA-C $_{8} /$ Water system was examined when the weight ratio, $R$, of $\mathrm{GFA}-\mathrm{C}_{8}$ to the total surfactants was changed keeping the total concentration of the surfactants at $5 \mathrm{wt} \%$. The formation of worm-like micelles was confirmed over a wide temperature range. Next, the effect of temperature on the rheological properties of the worm-like micelles was examined. From steady-flow viscosity measurements of the worm-like micelles, it was found that the zero-shear viscosity $\left(\eta_{0}\right)$ gave a maximum value more than 1,000 times greater than the minimum value in the temperature range $20-50^{\circ} \mathrm{C}$. Further, the temperature at which the maximum $\eta_{0}$ was observed decreased with increasing $R$ value. These results indicate that there is an optimal temperature at which the entanglement of worm-like micelles is at its greatest. From dynamic viscoelasticity measurements, it was shown that the viscoelastic behavior observed for the worm-like micelles was consistent with the Maxwell model, which is the basic model for a viscoelastic body. In addition, the plateau modulus $\left(G_{0}\right)$, which reflects the volume fraction of entangled worm-like micelles, gradually increased with increasing temperature, while the relaxation time $(\tau)$, which reflects the disentanglement time of the worm-like micelles, rapidly decreased with increasing temperature. From these results, it was clarified that, for a nonionic worm-like micelle, $\tau$ influences the change in $\eta_{0}$ more strongly than $G_{0}$.
\end{abstract}

Key words: worm-like micelle, polyoxyethylene phytosterol, glycerin fatty acid monoester, rheology, phase diagram, temperature

\section{INTRODUCTION}

Surfactants are critical materials in many industrial applications and are added to a number of commercial products such as medicines, cosmetics and foods. Surfactants are effectively amphipathic molecules, consisting of hydrophilic and hydrophobic groups, which in combination with water and oil phases interact to form a wide variety of molecular assemblies. Among these molecular assemblies, worm-like micelles are a very interesting example. Wormlike micelles are known to mutually entangle to form a gel with a high viscoelasticity, which arises due to the formation of a transient network structure. It is already known that worm-like micelles can be formed by the addition of aromatic salts such as sodium salicylate to cationic surfactants such as cetyltrimethylammonium bromide or cetylpyridinium chloride ${ }^{1-4)}$. Recently, Kunieda et al..$^{5-8)}$ reported that a worm-like micelle of high viscoelasticity was formed by mixing two types of nonionic surfactants with very different HLB (hydrophilic-lipophilic balance) values. For example, a worm-like micelle consisting of nonionic surfactants is formed by adding polyoxyethylene dodecyl ether, a hydrophobic molecule, to a spherical micellar solution of the hydrophilic molecule, polyoxyethylene cholesterol or polyoxyethylene phytosterol. Moreover, we have also reported the formation of a worm-like micelle in a mixed system consisting of polyoxyethylene phytos-

*Correspondence to: Kaname Hashizaki, College of Pharmacy, Nihon University, 7-7-1 Narashinodai, Funabashi, Chiba 274-8555, JAPAN E-mail: hashizaki.kaname@nihon-u.ac.jp

Accepted January 24, 2009 (received for review Jamuary 8, 2009)

Journal of Oleo Science ISSN 1345-8957 print / ISSN 1347-3352 online

http://www.jstage.jst.go.jp/browse/jos/ 


\section{K. Hashizaki, H. Taguchi and Y. Saito}

terols $\left(\mathrm{PhyEO}_{\mathrm{m}}\right)$ and glycerin fatty acid monoesters (GFA$\mathrm{C}_{\mathrm{n}}{ }^{9)}$. In this case, the formation of worm-like micelles takes place since $\mathrm{GFA}-\mathrm{C}_{\mathrm{n}}$ is solubilized within the palisade layer of a spherical or rod-like micelle consisting of $\mathrm{PhyEO}_{\mathrm{m}}$, and the interfacial curvature of the molecular assembly decreases as a result.

Raghavan et $a l^{4)}$ examined the influence of temperature on the rheological properties of worm-like micelles, and they clarified that worm-like micelles formed from ionic surfactants typically show an exponential decrease in zeroshear viscosity $\left(\eta_{0}\right)$ upon heating. However, there were few reports about the temperature behavior of worm-like micelles consisting of nonionic surfactants ${ }^{10,11)}$. In general, the heating of a nonionic surfactant system leads to phase separation because of dehydration of the head group; the temperature at which this phase separation occurs is called the cloud point. Polyoxyethylene nonionic surfactants, in particular, show a remarkably distinct cloud point. From this background, we looked to investigate the effects of temperature on the phase state and rheological behavior of worm-like micelles formed from nonionic surfactants. The control of the viscosity of worm-like micelles using temperature is expected to be useful in applications such as temperature-sensitive drug release system.

Towards this goal, we examined the effects of temperature on the rheological behavior of worm-like micelles formed from a nonionic surfactant system consisting of polyoxyethylene (10) phytosterol $\left(\mathrm{PhyEO}_{10}\right) /$ glyceryl monocaprylate $\left(\mathrm{GFA}-\mathrm{C}_{8}\right) /$ Water.

\section{EXPERIMENTAL}

\subsection{Materials}

Polyoxyethylene (10) phytosterol $\left(\mathrm{PhyEO}_{10}\right)$ was purchased from Nikko Chemicals Co., Ltd. (Tokyo, Japan) and was used as the hydrophilic nonionic surfactant. Glyceryl monocaprylate $\left(\mathrm{GFA}-\mathrm{C}_{8}\right)$ was a gift from Taiyo Kagaku Co., Ltd. (Mie, Japan) and was used as the hydrophobic nonionic surfactant. Distilled water for injection JP (Japanese Pharmacopoeia) was purchased from Otsuka Pharmaceuticals Co., Ltd. (Tokyo, Japan).

\subsection{Sample preparation and phase diagrams}

The required amounts of $\mathrm{PhyEO}_{10}, \mathrm{GFA}_{-} \mathrm{C}_{8}$ and water were placed in a screw cap bottle and mixed using a magnetic stirrer. The bottles were then thermostated at $25^{\circ} \mathrm{C}$ from a few days (for the micellar phase) to several weeks (for the liquid crystalline phase) to allow equilibration. Phase diagrams of the $\mathrm{PhyEO}_{10} / \mathrm{GFA}-\mathrm{C}_{8} /$ Water systems were obtained using visual observation through crossed polarizers and small angle X-ray scattering (SAXS) (NanoSTAR SSS, Bruker AXS Inc., WI, USA) analysis. Incidentally, a worm-like micellar solution is optically isotropic because it does not have a crystal structure; therefore, a characteristic pattern does not appear in the polarized light image. For the same reason, the scattering peak is not observed in the SAXS pattern of a worm-like micelle.

\subsection{Rheological measurements}

Steady and dynamic rheological measurements were performed using a rotational rheometer (HAAKE RS600, Thermo Fisher Scientific Inc., MA, USA) equipped with cone-plate geometry (two sizes: $60 \mathrm{~mm}$ and $35 \mathrm{~mm}$ diameters, each having a cone angle of $1^{\circ}$ and $4^{\circ}$ ) and a Peltierbased temperature control in the range of $20-50^{\circ} \mathrm{C}$. Moreover, a solvent trap was used in order to prevent evaporation. Dynamic rheological measurements were performed using a strain $(\gamma)$ value in the linear viscoelastic region.

\section{RESULTS AND DISCUSSION}

\subsection{Phase diagram of a PhyEO $_{10} /$ GFA-C $_{8} /$ Water system}

Figure 1 shows a temperature-composition phase diagram of $\mathrm{PhyEO}_{10} / \mathrm{GFA}_{-} \mathrm{C}_{8} /$ Water systems, in which the total surfactant concentration is fixed at $5 \mathrm{wt} \%$ and the weight ratio, $R$, of GFA-C 8 to the total surfactants is taken as the horizontal axis. A micellar phase (Wm) existed over a wide temperature range from 20 to $55^{\circ} \mathrm{C}$, and the formation of worm-like micelles was confirmed by the presence of a highly viscous region (shaded area) within the micellar phase (Wm). The formation mechanism of the worm-like

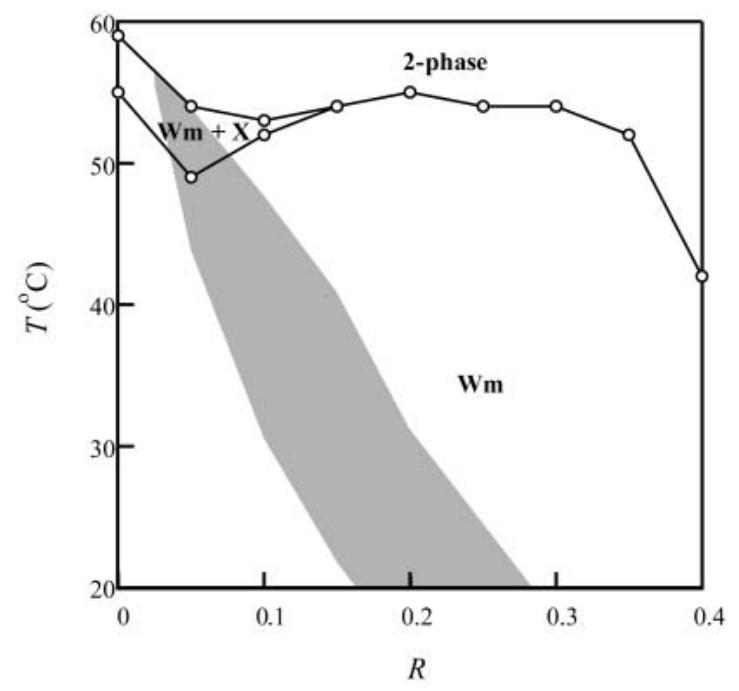

Fig. 1 Phase Diagram of a $5 \mathrm{wt} \% \mathrm{PhyEO}_{10} / \mathrm{GFA}^{\mathrm{C}} \mathrm{C}_{8} /$ Water System.

The notation Wm represents a micellar phase and $\mathrm{X}$ is an optically anisotropic liquid crystal phase of unknown structure ${ }^{8)}$. The region of high viscosity within the Wm phase is shown by the shaded area. 


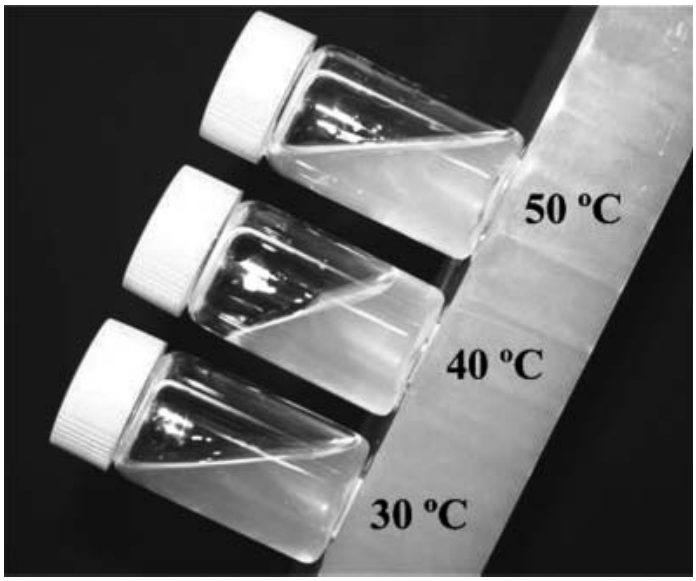

Fig. 2 Change in the Surface of Solutions at 30, 40, and $50^{\circ} \mathrm{C}$ of $5 \mathrm{wt} \% \mathrm{PhyEO}_{10} / \mathrm{GFA}_{-} \mathrm{C}_{8} /$ Water System $(R=$ $0.1)$ When Vials Are Tilted from an Upright Position.

micelles is explained in detail later in the text. The region in which worm-like micelles were formed moved from higher temperatures to lower temperatures as the $R$ value increased.

Figure 2 shows the appearance of solution surface at three different temperatures when vials of samples are tilted from an upright position. It can be seen that the surface inclines immediately after the vials are tilted for solutions except for the solution kept at $40^{\circ} \mathrm{C}$. All of the liquids were semitransparent and optically isotropic when left stationary, but showed a weak birefringence when moved. Moreover, the Wm and liquid crystalline $(\mathrm{X})$ phases were confirmed to coexist in the high temperature region corresponding to $R=0-0.15$ as shown in Fig. 1. This $\mathrm{X}$ phase was considered to be the same as an optically anisotropic liquid crystalline phase of unknown structure identified by Naito et al. in a $\mathrm{PhyEO}_{10} /$ Water system ${ }^{8}$. The borderline of transition between the $\mathrm{Wm}$ phase and the 2-phase region represents the cloud point curve in this system, and it was clarified that the cloud point decreased at higher $R$ values. A similar tendency was observed in the $10 \mathrm{wt} \%$ system.

\subsection{Effects of temperature on rheological behavior of a $\mathrm{PhyEO}_{10} / \mathrm{GFA}^{-\mathrm{C}_{8}} /$ Water system}

Figure 3 shows the relation between the zero-shear vis$\operatorname{cosity}\left(\eta_{0}\right)$ and temperature, where $\eta_{0}$ is given by the extrapolation of steady shear viscosity curve to zero shear rate. For $R=0.05$ to $0.15, \eta_{0}$ changes with temperature, reaching a maximum value more than 1,000 times greater than the minimum value. Furthermore, the temperature of the maximum $\eta_{0}$ value tended to decrease with increasing $R$ value. A similar tendency was observed for the $10 \mathrm{wt} \%$ system. These results indicate that there is an optimal

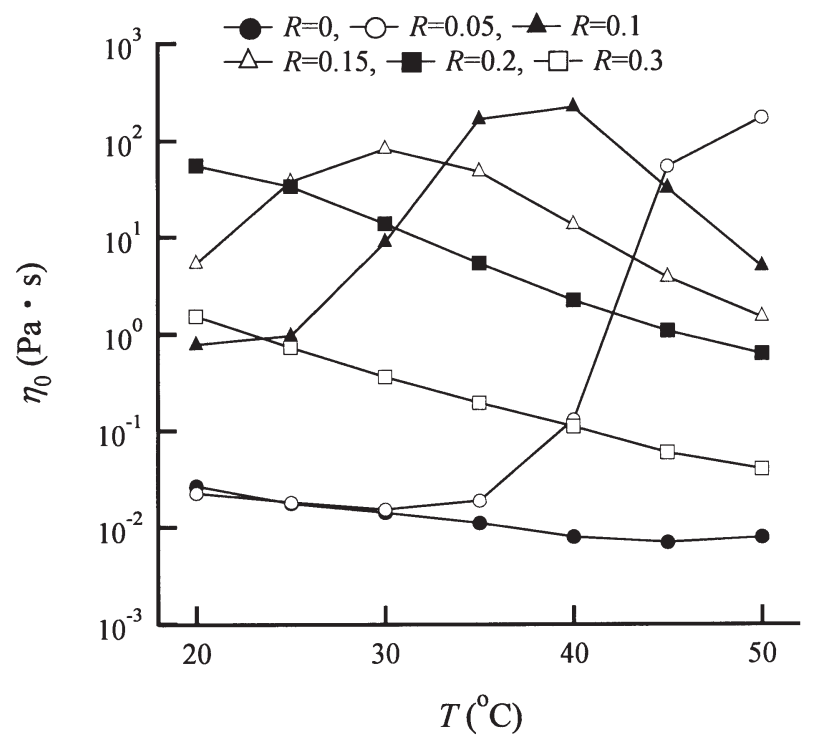

Fig. 3 Temperature Dependence of Zero-shear Viscosity $\left(\eta_{0}\right)$ at Different $R$ Values in $5 \mathrm{wt} \% \mathrm{PhyEO}_{10} / \mathrm{GFA}-$ $\mathrm{C}_{8}$ /Water System.

temperature at which the entanglement of worm-like micelles is at its greatest. Therefore, to examine the change in $\eta_{0}$ in greater detail, dynamic viscoelasticity measurements were performed at each temperature.

Figure 4 shows the variation in the storage modulus $\left(G^{\prime}\right)$ and loss modulus $\left(G^{\prime \prime}\right)$ as a function of the frequency $(\omega)$ for the $5 \mathrm{wt} \% \mathrm{PhyEO}_{10} / \mathrm{GFA}-\mathrm{C}_{8} /$ Water $(R=0.1)$ system as an example. Here, the $G^{\prime}$ and $G^{\prime \prime}$ values reflect the elasticity and viscosity, respectively. $G^{\prime}$ and $G^{\prime \prime}$ intersect at a certain $\omega$; at high frequencies the elasticity component is predominant $\left(G^{\prime}>G^{\prime \prime}\right)$ while at low frequencies the viscosity component is predominant $\left(G^{\prime \prime}>G^{\prime}\right)$. In general, the relaxation time of linear polymers has a wide distribution because the polymers disentangle via reptation (reptile-like motion) and the relaxation time is proportional to the third power of the molecular weight ${ }^{12-15)}$. On the other hand, the rheological behavior of worm-like micelles is decided by the balance between reversible breaking and reptation of the micelles, and single Maxwellian behavior is generally observed when the reversible breaking time is sufficiently shorter than the reptation time ${ }^{1)}$. In this situation, the relaxation time is given by the geometric mean of the breaking time and reptation time ${ }^{1)}$. As shown in Fig. 4a, the viscoelasticity behavior of each sample in the $20-30^{\circ} \mathrm{C}$ range was similar to that of polymer systems in which intertwining is insufficient, and it is thought that disentanglement of the worm-like micelles mainly takes place by reptation. On the other hand, the viscoelasticity behavior of each sample in the $35-50^{\circ} \mathrm{C}$ range, as shown in Fig. $4 \mathrm{~b}$, was similar to that expected for a Maxwell model and the worm-like micelles were observed to have a single relax- 

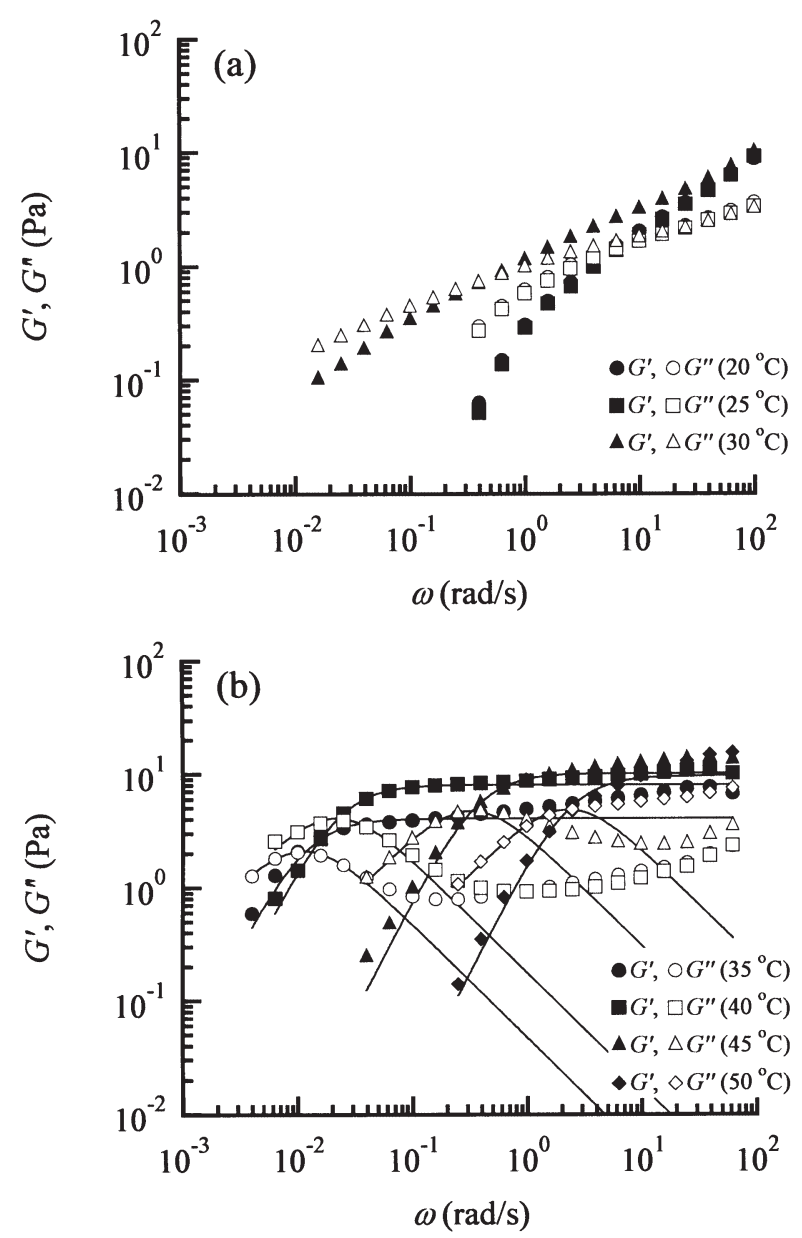

Fig. 4 Variation of $G^{\prime}$ and $G^{\prime \prime}$ as a Function of $\omega$ Obtained by Oscillation Frequency Sweep Measurements at Different Temperatures in $5 \mathrm{wt} \% \mathrm{PhyEO}_{10} / \mathrm{GFA}$ $\mathrm{C}_{8}$ /Water System $(R=0.1)$.

For clarity, the data are split into two plots: (a) 20, 25 , and $30^{\circ} \mathrm{C}$ and (b) $35,40,45$, and $50{ }^{\circ} \mathrm{C}$. Maxwellian fittings to the experimental data are shown by the solid lines.

ation time. From this, it is thought that disentanglement of the worm-like micelles mainly takes place by reversible breaking. Then, assuming that the worm-like micelles formed in this system follow a single Maxwell model, curve fitting can be performed based on Eqs. 1 and 2 .

$G^{\prime}=\frac{\omega^{2} \tau^{2}}{1+\omega^{2} \tau^{2}} G_{0}$

$G^{\prime \prime}=\frac{\omega \tau}{1+\omega^{2} \tau^{2}} G_{0}$

where $G_{0}$ is the plateau modulus reflecting the volume fraction of the entangled worm-like micelles ${ }^{1)}$ and $\tau$ is the relaxation time reflecting the disentanglement time of the worm-like micelles. The fitted curves are shown in Fig. 4b by solid lines. The rheological behavior of the worm-like micelles deviates from the Maxwellian fittings based on Eqs. 1 and 2 at high frequencies. This shows that the relaxation mode of the worm-like micelles formed in this system does not strictly apply to the single Maxwell model, and implies that a faster relaxation mode is occurring in addition to the reversible breaking. It is thought that this faster relaxation mode is related to micro-Brownian motion (i.e., the Rouse mode ${ }^{16)}$ ) of the worm-like micelle itself.

Figure 5 shows the variation in $G_{0}$ and $\tau$ as a function of temperature of the $5 \mathrm{wt} \% \mathrm{PhyEO}_{10} / \mathrm{GFA}^{-\mathrm{C}_{8}} /$ Water system. In the $R=0.1$ system shown in Fig. 3, in which $\eta_{0}$ showed a maximum in the vicinity of $40^{\circ} \mathrm{C}, G_{0}$ gradually increased with increasing temperature up to $40^{\circ} \mathrm{C}$, while $\tau$ rapidly decreased from $40^{\circ} \mathrm{C}$. On the other hand, in the $R=0.2$ system, in which $\eta_{0}$ gradually decreased over $20-50^{\circ} \mathrm{C}, G_{0}$ gradually increased with increasing temperature while $\tau$ rapidly decreased. These results indicate that the disentanglement time for the worm-like micelles rapidly decreases with increasing temperature despite an increase in volume fraction. We suggest that the worm-like micelles become breakable because of a reduction in the mechanical strength of the worm-like micelles as temperature increases. Furthermore, we sought to explain the reason for an increase in the volume fraction of the entangled worm-like micelles with increasing temperature. In general, the formation of worm-like micelles by nonionic surfactants takes place because the hydrophobic surfactant is solubilized within the palisade layer of a spherical micelle or rod-like micelle consisting of the hydrophilic surfactant, resulting

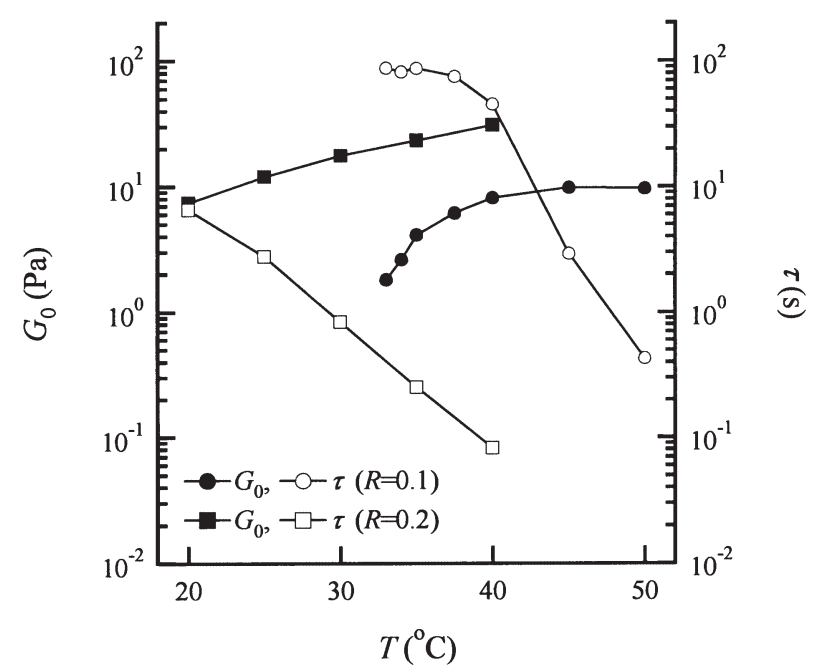

Fig. 5 Variation of $G_{0}$ and $\tau$ at Different Temperatures in 5 $\mathrm{wt} \% \mathrm{PhyEO}_{10} / \mathrm{GFA}-\mathrm{C}_{8} /$ Water System. 
in a decrease in the interfacial curvature of the molecular assembly. We propose that the formation of worm-like micelles with changing temperature follows a mechanism similar to that mentioned above. That is, it is well known that the hydrophobicity of a nonionic surfactant increases with increasing temperature because of dehydration of the head group upon heating. Therefore, it is thought that formation of the worm-like micelles is promoted because of the increase in hydrophobicity of $\mathrm{PhyEO}_{10}$ upon dehydration of the polyoxyethylene chain, resulting in a decrease in the interfacial curvature of the molecular assembly. When a sample follows a single Maxwell model, then $\eta_{0}$ can be defined as

$\eta_{0}=G_{0} \tau$

Here, $\eta_{0}$ is decided by the balance between the volume fraction and the disentanglement time of the entangled worm-like micelles. The $\eta_{0}$ values calculated using this equation were consistent with those obtained by steadyflow viscosity measurements (Fig. 3). The change in $\eta_{0}$ with temperature observed in Fig. 3 can be explained in terms of the relation between $G_{0}$ and $\tau$ as follows. That is, it is thought that $\eta_{0}$ increases with an increase in temperature not only because of an increase in the volume fraction of entangled worm-like micelles, but also because the worm-like micelles are not easily broken (large $\tau$ ). On the other hand, it is thought that $\eta_{0}$ decreases with increasing temperature because the worm-like micelles rapidly become more breakable (small $\tau$ ) as the volume fraction of the entangled worm-like micelles increases. The above results suggest that $\tau$ influences the change in $\eta_{0}$ of a nonionic worm-like micelle more strongly than $G_{0}$.

The control of the viscosity of worm-like micelles using the temperature variation and the surfactant composition suggests their possible application as a new gel base.

\section{ACKNOWLEDGEMENT}

This work was supported in part by the "High-Tech Research Center" Project for Private Universities, a matching fund subsidy from the Ministry of Education, Culture, Sports, Science and Technology (MEXT) of Japan, 2007 and a Nihon University Research Grant for 2007. Thanks are due to Ms. Nao Tamaki, Ms. Chinatsu Ohashi and Ms. Kana Sasaki for their technical assistance in the experimental work.

\section{References}

1. Cates, M.E.; Candau, S.J. Statics and dynamics of worm-like surfactant micelles. J. Phys.: Condens. Matter 2, 6869-6892 (1990).

2. Shikata, T.; Hirata, H.; Kotaka, T. Micelle formation of detergent molecules in aqueous media: viscoelastic properties of aqueous cetyltrimethylammonium bromide solutions. Langmuir 3, 1081-1086 (1987).

3. Imai, S.; Kunimoto, E.; Shikata, T. Viscoelastic behavior of surfactant threadlike micellar solutions-effects of additives 1. Nihon Reoroji Gakkaishi 28, 61-65 (2000).

4. Raghavan, S.R.; Kaler, E.W. Highly viscoelastic wormlike micellar solutions formed by cationic surfactants with long unsaturated tails. Langmuir 17, 300-306 (2001).

5. Acharya, D.P.; Kunieda, H. Formation of viscoelastic wormlike micellar solutions in mixed nonionic surfactant systems. J. Phys. Chem. B 107, 10168-10175 (2003).

6. Maestro, A.; Acharya, D.P.; Furukawa, H.; Gutierrez, J.M.; López-Quintela, M.A.; Ishitobi, M.; Kunieda, H. Formation and disruption of viscoelastic wormlike micellar networks in the mixed surfactant systems of sucrose alkanoate and polyoxyethylene alkyl ether. $J$. Phys. Chem. B 108, 14009-14016 (2004).

7. Naito, N.; Acharya, D.P.; Tanimura, J.; Kunieda, H. Rheological behavior of wormlike micellar solutions in mixed nonionic systems of polyoxyethylene phytosterol-polyoxyethylene dodecyl ether. J. Oleo Sci. 53, 599-606 (2004).

8. Naito, N.; Acharya, D.P.; Tanimura, J.; Kunieda, H. Phase behavior of polyoxyethylene phytosterol/polyoxyethylene dodecylether/water systems. J. Oleo Sci. 54, 7-13 (2005).

9. Hashizaki, K.; Tamaki, N.; Taguchi, H.; Saito, Y.; Tsuchiya, K.; Sakai, H.; Abe, M. Rheological behavior of worm-like micelles in a mixed nonionic surfactant system of a polyoxyethylene phytosterol and glycerin fatty acid monoester. Chem. Pharm. Bull. 56, 16821686 (2008).

10. Acharya, D.P.; Sharma, S.C.; Rodriguez-Abreu, C.; Aramaki, K. Viscoelastic micellar solutions in nonionic fluorinated surfactant systems. J. Phys. Chem. B 110, 20224-20234 (2006).

11. Varade, D.; Ushiyama, K.; Shrestha, L.K.; Aramaki, K. Wormlike micelles in Tween-80/ $\mathrm{C}_{m} \mathrm{EO}_{3}$ mixed nonionic surfactant systems in aqueous media. J. Colloid Interface Sci. 312, 489-497 (2007).

12. Doi, M.; Edwards, S.F. Dynamics of concentrated polymer systems. Part 1. Brownian motion in the equilibrium state. J. Chem. Soc., Faraday Trans. 274, 17891801 (1978).

13. Doi, M.; Edwards, S.F. Dynamics of concentrated polymer systems. Part 2. Molecular motion under flow. J. Chem. Soc., Faraday Trans. 274, 1802-1817 (1978).

14. Doi, M.; Edwards, S.F. Dynamics of concentrated poly- 


\section{K. Hashizaki, H. Taguchi and Y. Saito}

mer systems. Part 3. The constitutive equation. $J$. Chem. Soc., Faraday Trans. 274, 1818-1832 (1978).

15. Doi, M.; Edwards, S.F. Dynamics of concentrated polymer systems. Part 4. Rheological properties. J. Chem.
Soc., Faraday Trans. 275, 38-54 (1979).

16. Rouse, P.E. A theory of the linear viscoelastic properties of dilute solutions of coiling polymers. J. Chem. Phys. 21, 1272-1280 (1953). 